\title{
Diacronie
}

Studi di Storia Contemporanea

$N^{\circ} 33,1 \mid 2018$
Guerra e pace

\section{«Per una famiglia felice pace e lavoro»}

La propaganda al femminile del partito nuovo di Togliatti: simbologie e rituali del secondo dopoguerra

\section{Elisabetta Girotto}

\section{Q OpenEdition \\ 12 Journals}

\section{Edizione digitale}

URL: http://journals.openedition.org/diacronie/7235

DOI: 10.4000/diacronie.7235

ISSN: 2038-0925

Editore

Association culturelle Diacronie

Notizia bibliografica digitale

Elisabetta Girotto, « «Per una famiglia felice pace e lavoro» », Diacronie [Online], N 33, 1 | 2018,

documento 3, online dal 29 mars 2018, consultato il 10 décembre 2020. URL : http://

journals.openedition.org/diacronie/7235; DOI : https://doi.org/10.4000/diacronie.7235 


\title{
Diacronie
}

Studi di Storia Contemporanea

\section{$33,1 / 2018$}

Guerra e pace: declinazioni politiche, sociali e culturali del conflitto in età contemporanea

\section{«Per una famiglia felice pace e lavoro». La propaganda al femminile del partito nuovo di Togliatti: simbologie e rituali del secondo dopoguerra}

\author{
Elisabetta GIROTTO
}

Per citare questo articolo:

GIROTTO, Elisabetta, «"Per una famiglia felice pace e lavoro”. La propaganda al femminile del partito nuovo di Togliatti: simbologie e rituali del secondo dopoguerra», Diacronie. Studi di Storia Contemporanea : Guerra e pace: declinazioni politiche, sociali e culturali del conflitto in età contemporanea, 33, 1/2018, 29/03/2018,

URL: < http://www.studistorici.com/2018/03/29/girotto_numero_33/>

Diacronie Studi di Storia Contemporanea $\rightarrow$ http://www.diacronie.it

Rivista storica online. Uscita trimestrale.

redazione.diacronie@hotmail.it

Comitato di direzione: Naor Ben-Yehoyada - João Fábio Bertonha - Christopher Denis-Delacour - Maximiliano Fuentes Codera Anders Granås Kjøstvedt - John Paul Newman - Deborah Paci - Niccolò Pianciola - Spyridon Ploumidis - Wilko Graf Von Hardenberg

Comitato di redazione: Jacopo Bassi - Luca Bufarale - Gianluca Canè - Luca G. Manenti - Fausto Pietrancosta - Alessandro Salvador - Matteo Tomasoni - Luca Zuccolo 


\title{
3/ «Per una famiglia felice pace e lavoro». La propaganda al femminile del partito nuovo di Togliatti: simbologie e rituali del secondo dopoguerra
}

\author{
Elisabetta GIROTTO
}

Attraverso l'analisi dei linguaggi della propaganda politica si individuano simboli e rituali che caratterizzarono la comunicazione politica del partito nuovo di Togliatti. Centrale è il movimento femminile dell'Udi nella sua azione di assistenza e previdenza all'infanzia e alla famiglia. D'altro canto in un paese che come l'Italia appare caratterizzato dalla mancanza di un sentimento condiviso di appartenenza nazionale, far appello ai valori familiari risultò una formula vincente. Essenziale risulta lo studio del linguaggio simbolico che permette di decodificare i dispositivi attraverso i quali si definisce lo spazio pubblico della politica. Pertanto l'analisi dell'uso pubblico della famiglia da parte del Pci è il focus di questo lavoro volto a individuare immagini, gesti ed emblemi che concorrono ad articolare un universo di simboli che condensano funzioni pragmatiche e significati semantici, progetti razionalisti di utopie egualitarie e manifestazioni rituali.

\section{Introduzione}

Nel linguaggio politico del secondo dopoguerra, in un paese che come l'Italia appare caratterizzato dalla mancanza di un sentimento condiviso di appartenenza nazionale, far appello ai valori familiari risulta senza dubbio una formula vincente. Chiamando in causa la famiglia, la sua salvaguardia e il suo consolidamento, si è certi di cogliere nel segno, se si vuol "capitalizzare" a proprio vantaggio un elemento primario dell'universo mentale degli italiani e soprattutto delle italiane. Il momento si prospetta per di più singolarmente propizio. Ad esaltare la dimensione del privato, concepita nella sua accezione solidaristica, avevano contribuito i lunghi anni di guerra e la liberazione dalle invadenze dell'occhiuto tentativo di controllo totalitario del regime, mentre lo straordinario prestigio acquisito sul piano politico dalla Chiesa dopo l'8 settembre, e l'intensificarsi del sentimento religioso, di fronte alla percezione della precarietà dell'esistenza, contribuivano a rafforzarne la tradizionale struttura patriarcale. Tuttavia è anche vero che la 
partecipazione femminile nella Resistenza (sia quella armata che quella civile) aveva contribuito a mettere in crisi la visione tradizionale della donna consolidatasi durante il regime fascista ${ }^{1}$.

Era più che prevedibile, in altra parole, che l'“uso pubblico" della famiglia finisse col circoscrivere uno dei terreni privilegiati su cui si sarebbe combattuta la battaglia politica dal 1946 in poi. Protagonista incontrastata del linguaggio democristiano ${ }^{2}$, sarà la dimensione parentale, non quella individualistica, a rappresentare il terreno comune di espressione degli appelli elettorali, a innervare le campagne propagandistiche e la rappresentazione iconografica e simbolica della comunicazione politica del dopoguerra ${ }^{3}$. Non a caso, nel Messaggio della Democrazia cristiana agli italiani, in occasione delle elezioni dell'Assemblea Costituente ${ }^{4}$, si sollecita un voto destinato a «salvare la nostra civiltà», cioè i valori «dell'unità e indissolubilità del Matrimonio, della prosperità della famiglia, dell'educazione cristiana dei figli» ancor prima di fare riferimento al «riscatto del lavoro e della proprietà privata» ${ }^{5}$.

Immagini della famiglia d'altra parte imperano ovunque. Chi passava per le strade, soprattutto, ma non solo, in tempo di elezioni, veniva costantemente assalito da manifesti popolati da ritratti di donne e bambini: ora mesti e macilenti, ora paffuti e radiosi.

Così la partita destinata a giocarsi con le nuove regole della democrazia sembra tradursi, sotto molti punti di vista, in una gara per il primato nella "difesa" delle famiglie italiane dalle minacce che la assalgono sul fronte nemico: una gara particolarmente serrata sul fronte dell'elettorato femminile, dal quale peraltro sarebbe dipeso in buona parte l'esito delle urne. E qui la Dc partiva con un vantaggio incommensurabile. Come se non bastasse l'imprimatur della tradizione cattolica, sacerdoti e attiviste si mobilitano in massa per suggerire adesso un voto "per il Bene contro il Male ${ }^{6}$, a salvaguardia del sacramento del Matrimonio insidiato da presunte trame

\footnotetext{
${ }^{1}$ GINSBORG, Paul, Famiglia Novecento, Torino, Einaudi, 2013, pp. 250-253, 258-260; DE GRAZIA, Vittoria, Le donne del regime fascista, Venezia, Marsilio, 1993; GIROTTO, Elisabetta, «Donne in divisa. Donne, politica e famiglia nei cinegiornali Luce degli anni Trenta», in Officina della Storia, 1/2018, URL:

< https://www.officinadellastoria.eu/it/2018/01/04/donne-in-divisa-donne-politica-e-famiglia-nei-cinegiornaliluce-degli-anni-trenta/ > [consultato il 14 febbraio 2018].

2 URSO, Simona, Dalla comunità naturale allo spazio politico: donne democristiane a Torino 1946-1990, in SILVESTRINI, Maria Teresa, SIMIAND, Caterina, URSO, Simona (a cura di), Donne e politica. La presenza femminile nei partiti politici dell'Italia repubblicana, 1945-1990, Milano, Franco Angeli, 2005, pp. 27-128, pp. 29-30, p. 39 e passim.

${ }^{3}$ GIROTTO, Elisabetta, «Politics and Media. The audiovisual representation of family made by the Pci and the Dc during the fifties», in Memoria e Ricerca, XXII, 47, 3/2014, pp. 151-182.

4 Sulla visione togliattiana della famiglia nel dibattito all'Assemblea Costituente cfr., fra gli altri, CAPORELLA, Vittorio, «La famiglia nella Costituzione italiana. La genesi dell'articolo 29 e il dibattito della Costituente», in Storicamente, VI, 9, 2010, URL: < https://storicamente.org/famiglia_costituzione_italiana > [consultato il 14 febbraio 2018]; LUSSANA, Fiamma, «Famiglia e indissolubilità del matrimonio nel dibattito all'Assemblea Costituente», in Studi Storici, LV, 2/2014, pp. 495-519.

${ }^{5}$ Messaggio della Democrazia cristiana agli italiani, cit. in RIDOLFI, Maurizio, TRANFAGLIA, Nicola, 1946. La nascita della Repubblica, Roma-Bari, Laterza, 1996, p. 104.

${ }^{6}$ SALVI, C., «Una calamità dopo tante altre. Dibattiti sul divorzio», in Il settimanale della donna, 7, 7-10 febbraio 1946; TITTONI, Maria, «La donna e le elezioni. Voteremo per chi garantirà al popolo italiano pane, lavoro, proprietà per tutti e rispetto per la religione», in Il settimanale della donna, 14, 7 aprile 1946.
} 
divorziste. Più che di un invito del resto si trattava di una vera e propria imposizione, visto che chi non votava per il partito di Dio - si andava ripetendo - commetteva peccato mortale ${ }^{7}$.

Insomma l'arma del duello l'aveva scelta la Democrazia cristiana, e uno sfidante che in passato aveva a lungo disquisito sull'estinzione della famiglia arrivava all'appuntamento praticamente disarmato. Solo un concorrente abile quanto duttile e instancabile come Togliatti poteva accettare la sfida, orchestrando un vero e proprio capovolgimento del patrimonio ideologico del "vecchio" Pci incentrato sul concetto sostanzialmente anaffettivo della coppia di «rivoluzionari di professione», per farlo germogliare a beneficio delle elettrici in un'icona della famiglia tradizionale quale cellula primaria del comunismo. Un vero e proprio capolavoro nell'arte della propaganda, il suo, nel quale le nuove istanze staliniane di rivalutazione dell'istituto familiare sarebbero arrivate alle orecchie degli italiani nella traduzione gramsciana ${ }^{8}$.

Poco importava se il prezzo da pagare era quello di cavalcare tutti i clichés più sperimentati in tema di costruzione delle identità di genere, riproponendo puntualmente i vecchi stilemi ai quali si dava per scontato che le italiane restassero ancorate "per natura". Rivolgendosi a loro il Pci promette «la pace, il lavoro e la ricostruzione delle famiglie sconvolte e distrutte, [...] [la salvezza] della gioventù e dell'infanzia dalla depravazione, dal deperimento e da nuove guerre» e solo in seconda istanza la parità di diritti ${ }^{9}$. Così, per rafforzare l'incisività del messaggio, la parola d'ordine imposta dal partito in occasione dell'organizzazione della Settimana della compagna, indetta dopo la convocazione della I Conferenza delle donne comuniste, nel 1945, era stata: «Per la protezione delle famiglie italiane» ${ }^{10}$.

Il segretario aveva ben chiaro che era necessario «avere le donne dalla nostra parte». E per avvicinarsi a loro bisognava parlare la loro lingua. L'orditura dello spartito preelettorale del nuovo partito di massa sarebbe stata tessuta così con gli elementi del tipico frasario cattolico.

Alla crociata anticomunista indetta da Pio XII si reagisce in altre parole con i toni manierati e zuccherosi della precettistica parrocchiale, ritorcendo contro "il nemico" schierato sul campo di

7 DAU NOVELLI, Cecilia, Il movimento femminile della Democrazia cristiana dal 1944 al 1946, in MALGERI, Francesco (a cura di), Storia della Democrazia cristiana, vol. III, 1955-1968, Roma, Edizioni Cinque Lune, 1988, p. 340.

${ }^{8}$ Sul Pci cfr. VENTRONE, Angelo, «La liturgia politica comunista dal '44 al '46», in Storia contemporanea, XIV, 4/1992, pp. 779-836; si veda inoltre ID., Forme e strumenti della propaganda di massa nella nascita e nel consolidamento della repubblica, in RIDOLFI, Maurizio (a cura di), Propaganda e comunicazione politica, Milano, Mondadori, 2004, pp. 209-232. Interessanti spunti di riflessione provengono dal recente studio di RIDOLFI, Maurizio, Italia a colori. Storia delle passioni politiche dalla caduta del fascismo ad oggi, Firenze, Le Monnier, 2015, pp. 30-55, dove si riflette sulle varie fasi che caratterizzarono la vita politica italiana del secondo dopoguerra attraverso un approccio politico e socio-culturale.

${ }^{9}$ Cfr. Le donne italiane devono votare per $i$ comunisti e per la Repubblica, Istituto Gramsci, Archivio Partito Comunista, fasc. Monarchia e repubblica, cit. in RIDOLFI, Maurizio, TRANFAGLIA, Nicola, 1946, cit., p. 205.

${ }^{10}$ PASTI, Daniela, I comunisti e l'amore, Milano, Edizioni de L'Espresso, 1979. 
battaglia, la resa delle armi, quell'arrendevolezza della pietas cristiana che dell'armamentario pedagogico del linguaggio cattolico aveva sempre rappresentato l'arma più forte ${ }^{11}$.

Visto che con la religione non si può competere - e questo l'aveva capito anche Mussolini l'unica possibilità di vittoria stava nell'accaparrare alla propria parte politica il suo enorme potenziale suggestivo, cercando di rivolgere contro "il partito di Cristo" le sue stesse armi. «La messa è in parte un comizio»: i comunisti non dispongono di strumenti altrettanto sperimentati per far centro nelle menti semplici delle donne italiane, osservava Togliatti, ma non per questo dovevano cedere alla Dc il monopolio di un insuperabile apparato propagandistico ${ }^{12}$.

\section{2. «Punisci noi peccatori, ma salva i bambini»}

Chi potrebbe immaginare, a prima vista, di trovarsi di fronte ad uno slogan dell'Udi? ${ }^{13}$ In realtà proprio il tema della «Salvezza dell'infanzia» costituisce uno degli assi portanti della "catechesi" comunista. E su questo precetto si impernia la campagna di rassicurazione delle italiane delegata alle compagne, il cui primo compito era quello di sfatare le accuse mosse al Pci e «soprattutto alle comuniste: [di essere] contro la famiglia, la proprietà e la religione» ${ }^{14}$. «Salvare l'infanzia minacciata dalla malnutrizione, la riapertura delle scuole, la creazione di colonie estive, un'equa distribuzione dei soccorsi UNRRA, l'offerta di pacchi dono ai bambini per le feste di fine anno»: questi si prospettavano come gli obiettivi più urgenti che l'organizzazione femminile del Pci doveva prefiggersi - dichiarava Rita Montagnana ${ }^{15}$. Ed Egle Gualdi le faceva eco ribadendo come il compito fondamentale delle donne comuniste (che così facendo avrebbero anche «fatto un grande passo avanti per l'emancipazione femminile») fosse quello di risolvere «i principali

\footnotetext{
${ }^{11} \mathrm{Cfr}$. l'articolo di fondo «Salvare la famiglia italiana», in Noi donne, 20 luglio 1946.

${ }^{12}$ TOGLIATTI, Palmiro, «È stato giusto dare il voto alle donne?», discorso pronunciato alla riunione delle attiviste di Roma, Roma, 13 maggio 1953, in ID., L'emancipazione femminile, cit., p. 78. Sul rapporto fra il Pci e la famiglia esemplare il lavoro di BELLASSAI, Sandro, La morale comunista. Pubblico e privato nella rappresentazione del Pci 1947-1956, Roma, Carocci, 2000.

${ }^{13}$ Cfr. «Pietà per tutti i bimbi», in Noi donne, 19 Settembre 1946, interamente dedicato ai bambini, che «possano crescere sani, abbiano nutrimento, vivano in case sane, abbiano assistenza igienica e non siano esposti ai pericoli della strada». Cfr. inoltre «Per la salvezza dell'infanzia e della gioventù», in Noi donne, 1 agosto 1946; MONTAGNANA, Rita, «Tetto, pane, vestirti, scuole per i nostri bimbi», in Noi donne, 25 ottobre 1946.

${ }^{14}$ «Le donne italiane hanno lottato ieri per la Liberazione, esse vogliono oggi contribuire alla ricostruzione del paese», in L'Unità, 3 giugno 1945. Non a caso, ancor più della Democrazia Cristiana, il vero avversario del Pci sarebbe stato la Chiesa. Cfr. MICCOLI, Giovanni, Cattolici e comunisti nel secondo dopoguerra. Memoria storica, ideologia e lotta politica, in MICCOLI, Giovanni, NEPPI MODONA, Guido, POMBENI, Paolo (a cura di), La grande cesura. La memoria della guerra e della resistenza nella vita europea del dopoguerra, Bologna, Il Mulino, 2001, pp. 31-88, p. 83.

${ }^{15}$ MONTAGNANA, Rita, «Una vittoria della democrazia», in L'Unità, 1 novembre 1945.
} 
problemi della vita comunale» laddove al primo posto si nominava «l'assistenza ai bambini e alle gestanti» e in seconda istanza «i problemi alimentari, la luce, l'acqua, il gas» ${ }^{16}$.

Se le parole d'ordine del linguaggio femminile del partito erano quindi famiglia, moralità e soprattutto centralità dell'infanzia, d'altra parte la costruzione degli slogan di propaganda era volta a sottolineare le opere che in realtà furono messe in pratica.

La premessa, del resto, del progetto di ricostruzione familiare del dopoguerra era già implicita nella stessa costituzione dell'Udi (che in quanto organizzazione di tutte le donne con il Pci non aveva niente a che fare - si continuava a ripetere dalla Segreteria del Pci) e nella definizione della sfera separata di azione delle compagne, tutta incentrata nel campo dell'assistenza, prospettata da Togliatti appena messo piede a Salerno.

Come ricorda Maria Casalini:

Il compito assegnato al collateralismo femminile nell'ambito della strategia comunista era quindi tutt'altro che secondario, ma anche particolarmente delicato. Fra le donne, il cui orientamento politico avrebbe rappresentato la vera grande incognita delle elezioni dal 1946 in poi, doveva compiersi un'opera di convinzione, imperniata più su segnali subliminali che sull'indottrinamento ideologico ${ }^{17}$.

La strategia di avvicinamento all'elettorato femminile doveva procedere quindi in maniera "trasversale", articolandosi attorno ad elementari parole d'ordine che andassero incontro alle loro esigenze quotidiane, in modo che il voto a sinistra fosse l'espressione più immediata dall'aspirazione a migliorare la qualità della vita dell'intera famiglia ${ }^{18}$.

Le esigenze cui si cerca di andare incontro risultano così le più varie, ma al primo posto resta sempre e comunque la necessità di alleviare le sofferenze dei più fragili e indifesi: i bimbi, vittime designate del «rachitismo e di tubercolosi» dilaganti ${ }^{19}$. Già nel 1944 la prima iniziativa dell'Udi era stata interamente dedicata a loro, in occasione della celebrazione del rito del «Natale della liberazione» a Reggio Calabria, Catania, Cagliari, Ancona, dove insieme ai regali si erano distribuiti i grembiulini confezionati con le bandiere e le camicie nere del Fascio ${ }^{20}$. Ma era stata soprattutto la celebrazione fiorentina, dove si sarebbe addobbato per i bambini l'«Albero di Natale della Libertà», a restare memorabile. Sarà la prima iniziativa di solidarietà di massa per

\footnotetext{
${ }^{16}$ GUALDI, Egle, «La donna italiana e le elezioni amministrative», in L’Unità, 6 giugno 1945.

${ }^{17}$ CASALINI, Maria, Le donne della sinistra, Roma, Carocci, 2005, p. 139.

${ }^{18}$ Ibidem.

${ }^{19}$ «La mozione conclusiva», in Noi donne, 31 ottobre -15 novembre 1945.

${ }^{20}$ LUSSANA, Fiamma, 1944-1945: Togliatti, "la via italiana", le donne, in BONACCHI, Gabriella, DAU NOVELLI, Cecilia (a cura di), Culture politiche e dimensioni del femminile nell'Italia del '900, Soveria Mannelli, Rubbettino, 2010 , pp. 137-167, p. 150. Sul "Natale di liberazione" cfr. "Rapporto di Rita Montagnana al I Congresso nazionale dell'Udi», cit. in MICHETTI, Maria, REPETTO, Margherita, VIVIANI, Luciana, Udi: laboratorio di politica delle donne: idee e materiali per una storia, Soveria Mannelli, Rubbettino, 1998, p. 31.
} 
l'infanzia: una sorta di prova generale della serie di riti collettivi che avrebbero costellato il lungo dopoguerra, durante la quale si sperimenta per la prima volta la rappresentazione della "società pacificata del futuro". Così Dina Ermini ricorda l'iniziativa:

Nonostante il clima caotico di quel periodo, il lavoro fu organizzato in modo esemplare: attraverso un censimento strada per strada dei bambini da assistere - ben più numerosi del previsto -, al quale seguì una raccolta capillare di tutto: indumenti, giocattoli, libri, generi alimentari, in modo da far fronte alle più diverse necessità, mentre ovunque si organizzavano tombole, lotterie, feste, concerti, film, si raccoglievano prenotazioni per i bambini nei ristoranti della città, e fu organizzata persino, con il concorso del maestro Vuotto, la rappresentazione di un'opera al teatro Comunale a beneficio del Natale popolare. I risultati superarono ogni previsione: invece di 5000 pacchi [...] ne furono distribuiti 15.000 [...]. Gli aiuti furono dati valutando le esigenze delle famiglie e la condizione dei bambini, non escludendo dopo animate discussioni per vincere le immaginabili resistenze - degli orfani dei fascisti o i bimbi di gente scappata al nord nella repubblica di Salò: nessun bambino doveva pagare per colpe non sue o sentirsi abbandonato il giorno di Natale ${ }^{21}$.

Da quel momento in poi le iniziative per i bambini si sarebbero susseguite con regolarità ${ }^{22}$. Per loro ci sarebbe sempre stato spazio nel calendario degli appuntamenti delle compagne, puntualmente corredate dalla programmazione di recite e gare per i piccoli, con distribuzione di libri e giocattoli ${ }^{23}$. Per il resto, già a partire dal 1944 e durante tutto dell'anno successivo, colonie estive, scuole, asili, aiuti invernali avrebbero rappresentato l'attività primaria delle compagne, in continuità con la tradizione assistenziale dei Fasci femminili ${ }^{24}$. Tant'è che il manifesto elettorale del 2 giugno 1946 apparso su «Noi donne» recitava «BIMBI: ciò che l'Udi ha fatto per l'infanzia (Alimentazione, asili, doposcuola, colonie, Natale Udi, [e, dulcis in fundo,] accoglienza in regioni salubri e case ospitali per 25.000 bambini)».

Proprio la campagna di ospitalità ai bambini poveri del Sud, offerta dalle famiglie dei compagni meno disagiati del Centro Nord appariva in realtà la punta di diamante, a livello propagandistico, della politica assistenziale del $\mathrm{Pci}^{25}$. Avviata nell'autunno del 1945, l'iniziativa a favore della

\footnotetext{
${ }^{21}$ MINELLA, Angiola, SPANO, Nadia, TERRANOVA, Ferdinando (a cura di), Cari bambini vi aspettiamo con gioia... Il movimento di solidarietà popolare per la salvezza dell'infanzia negli anni del dopoguerra, Milano, Teti Editore, 1980 , p. 29.

${ }^{22}$ Sull'ospitalità dei bambini poveri, che l'Udi organizza fra il 1945-46 e negli anni successivi, stimolanti gli studi di RINALDI, Giovanni, I treni della felicità. Storie di bambini in viaggio tra due Italie, Roma, Ediesse, 2009; PIVATO, Stefano, I comunisti mangiano i bambini. Storia di una leggenda, Bologna, Il Mulino, 2013, pp. 135-145. ${ }^{23} \mathrm{Cfr}$. in proposito LUSSANA, Fiamma, 1944-1945: Togliatti, cit., p. 163.

${ }^{24}$ Sui partiti assistenziali, cfr. VENTRONE, Angelo, Forme e strumenti della propaganda di massa nella nascita e nel consolidamento della repubblica (1946-1958), in RIDOLFI, Maurizio (a cura di), Propaganda e comunicazione politica, Storia e trasformazioni nell'età contemporanea, Milano, Mondadori, 2004, pp. 209-232, p. 220.

${ }^{25}$ Cfr. MAFAI, Miriam, L'apprendistato della politica. Le donne italiane nel dopoguerra, Roma, Editori Riuniti,
} 
«salvezza dell'infanzia» avrebbe raggiunto il massimo sviluppo tra il 1946 e il 1947, per poi riprendere dopo il voto del 18 aprile del 1948. Si era trattato di un'impresa imponente che aveva subito visto partire ben quattromila bambini di Cassino e diecimila piccoli napoletani; poi sarebbe stata la volta dei bambini lucani. E può risultare interessante osservarla più da vicino, perché al suo interno si intrecciano stili e contenuti propagandistici diversi, la cui mistura appare il frutto di una accurata orchestrazione, destinata ad incassare una risonanza pubblicitaria straordinaria.

La ricetta, in perfetto stile nazional popolare, sul piano simbolico vuol soprattutto offrire una testimonianza delle infinite potenzialità implicite nella sostituzione del concetto di solidarietà a quello della tradizionale "carità delle dame»" . Una solidarietà che nasce dal basso e proprio dal cuore di quelle famiglie che la fede comunista ha reso infinitamente più aperte e generose di quella cattolica, a dispetto delle calunnie degli avversari politici, pronti a spargere la voce che i bambini, una volta caricati sui treni, sarebbero stati tutti deportati in Russia ${ }^{27}$.

Ma gli obiettivi del progetto comunista erano ancora più ambiziosi. L'esibizione del ruolo sociale della famiglia (comunista) risultava infatti strettamente legata al tema dell'unità nazionale. Non a caso, in occasione della campagna organizzata dal Comitato per la salvezza dei bambini di Napoli, rispolverando echi dal sapore risorgimentale, si era lanciato un «appello ai fratelli delle altre regioni d'Italia» ${ }^{28}$, mentre le bandiere rosse cedevano il passo al tricolore e le note dell'Internazionale a quelle dell'inno di Mameli ${ }^{29}$.

L'effetto propagandistico dell'iniziativa, a cui viene data ampia risonanza sulla stampa e alla radio, avrebbe trovato un primo sbocco rituale nello sfoggio delle onorificenze tributate al treno della solidarietà, nel momento in cui il convoglio carico di bambini attraversa le diverse stazioni. Ricorda Dina Ermini:

La cosa più commovente fu l'accoglienza della folla che ci attendeva al passaggio: in ogni stazione intermedia erano organizzati posti di ristoro con latte caldo, biscotti, frutta; nella stazione di arrivo ci accoglievano le autorità, il sindaco con la fascia tricolore, la folla plaudente, mentre la banda locale suonava lungo il treno carico di bambini, addobbato a festa con il tricolore e le bandiere dei comuni. A Reggio Emilia avevano addirittura eretto un palco

1979, pp. 137-141.

${ }^{26}$ FIORE, M. e JACOVELLO, A., nel loro opuscolo Aiutiamo i bambini di Napoli, (s. 1., 1946, p. 3) tengono a precisare che il cardinal Ascalesi, arcivescovo di Napoli, aveva negato la propria collaborazione all'iniziativa "perché la Curia aveva la propria rete assistenziale». Per i temi di "assistenza, non beneficenza" cfr. FLOREANINI, Gisella, «Una donna nel governo dell'Ossola», in Rinascita, 4/1955; sul tema interessanti spunti di riflessione provengono da CASALINI, Maria, op. cit., p. 94

${ }^{27}$ Ibidem, p. 65.

${ }^{28}$ MACCHIAROLI, Angelo, Un'esperienza popolare del dopoguerra per la salvezza dei bambini di Napoli, Napoli, Arte grafica, 1979.

${ }^{29}$ Direttamente dalla Direzione del partito era partita la raccomandazione di sostituire il più possibile il tricolore alle bandiere rosse: in proposito cfr. VENTRONE, Angelo, La cittadinanza repubblicana. Forma partito e identità nazionale alle origini della democrazia italiana (1943-1948), Bologna, Il Mulino, 1996, pp. 181-183. 
speciale dentro la stazione con tutte le rappresentanze cittadine mentre fuori sul piazzale si allungava la fila dei pullman imbandierati, con le insegne dei comuni ospitanti ${ }^{30}$.

Al rito del viaggio di andata sarebbe corrisposto poi quello del ritorno, fino alla coreografia finale quando il treno della bontà, che carico di striscioni aveva attraversato mezza Italia, avrebbe restituito i bimbi ai genitori: memorabile quella tributata nel luglio del 1947 ai piccoli napoletani, rifocillati e messi letteralmente all'ingrasso dalle famiglie dei compagni del Nord (quelle stesse famiglie settentrionali di cui si continuano a lamentare in altre sedi le difficilissime condizioni di vita). Per celebrare l'evento, alla festa di Piedigrotta, insieme agli altri sarebbe infatti sfilato il carro allegorico Nord-Sud: un simbolico ponte attraversato da un treno in movimento, la cui rotaie erano uscite «dal laminatoio dell'Ilva come le fettuccine dalla macchina della pasta», che univa il Vesuvio alle Due Torri, Napoli a Bologna e alle altre città che avevano ospitato i bambini. E lungo tutto il percorso della sfilata, da piazza Carlo V, per Forìa, il Museo, Toledo, San Ferdinando, via Caracciolo, fino a Mergellina:

$\mathrm{Fu}$ un applauso senza interruzione - commenta uno dei membri del Comitato organizzativo per la grandiosità del carro, la bellezza dei bambini, e delle accompagnatrici, lo splendore dei costumi e anche perché della folla facevano parte centinaia di famiglie dei bambini che erano stati ospiti del comitato ${ }^{31}$

Niente di più evidente dunque di come il tema della festa, l'allegria del riconoscersi come una comunità coesa, rappresenti un elemento ineliminabile della liturgia comunista. Una liturgia senza dubbio confezionata con sapienza fin nei minimi dettagli, ma pur sempre incapace di far breccia nel muro di diffidenza alimentato dalla propaganda cattolica. I bambini - non si sarebbero stancati di ripetere di lì a poco i Comitati civici - i comunisti potevano anche vezzeggiarli, ma alla fine se li sarebbero mangiati comunque!

\section{Il socialismo è gioia}

Per rendersi conto di quanto la propaganda comunista avesse investito sul "logo" della mitezza basta guardare le immagini sui manifesti elettorali: famiglie felici, con padre madre

\footnotetext{
${ }^{30}$ Testimonianza di ERMINI, Diana, in MINELLA, Angiola, SPANO, Nadia, TERRANOVA, Ferdinando (a cura di), op. cit., p. 47.

${ }^{31}$ Sulla scia dell'entusiasmo per i successi ottenuti, in linea con la strategia del radicamento capillare della propaganda nel tessuto urbano dal comitato per gli aiuti ai bambini di Napoli sarebbero poi sorti altri sottocomitati, finanziati, oltre che da lotterie rionali e sottoscrizioni, da una straordinaria mostra di quadri - con tele di Craemer, de Pisis e Mafai - i cui introiti erano destinati alla creazione di «mense gratuite per i figli del popolo».
} 
(apparentemente di ceto medio) e figli (generalmente due) che avanzano ottimisti verso il futuro radioso che li attende; contadine spensierate e sorridenti che recano in mano mazzi di fiori rossi, mamme con bambini in braccio che sventolano bandiere tricolori ${ }^{32}$.

Non c'è dubbio che

L'immagine femminile di sinistra, in altre parole, è sempre depurata di qualsiasi aggressività e in ciò si differenzia completamente da quella delle madri eroiche dei manifesti democristiani, affollati da raffigurazioni di donne ora armate di pugnali recanti la didascalia «voto cristiano», pronte a difendere i figli dagli attacchi di mostri rosso vestiti, ora impegnate ad uccidere serpenti divorzisti inneggianti il libero amore per salvare i propri bambini dal bolscevismo ${ }^{33}$.

A colpo d'occhio, insomma, il contrasto tra la violenza delle immagini dei manifesti democristiani e la pacatezza comunista non potrebbe essere più palese, a testimonianza di come l'esibizione d'immagini rassicuranti apparisse evidentemente al Pci il miglior antidoto contro i veleni con cui gli avversari tentavano di intossicare il clima politico del dopoguerra ${ }^{34}$. E sulla stessa lunghezza d'onda, nel palinsesto della orchestrazione degli appuntamenti rituali del movimento operaio, oltre al recupero della tradizione socialista della convivialità ${ }^{35}$, si inseriva un chiaro richiamo ai modelli ricreativi che gli italiani si erano abituati a condividere prima della guerra $^{36}$. Il comizio era destinato a rimanere il momento culminante di qualsiasi manifestazione politica, ma per trasformare l'iniziativa in un vero e proprio evento era necessario inserire una nota gioiosa e allo stesso tempo ricca di agganci con gli elementi tradizionali della mentalità collettiva. Di questo Giancarlo Pajetta era fermamente convinto, soprattutto se si intendeva riuscire a coinvolgere nelle manifestazioni del partito anche «le donne, i vecchi, i bambini»». È soprattutto a loro che si rivolge del resto il richiamo delle Feste dell'Unità, inizialmente concepite

\footnotetext{
${ }^{32}$ Per una analisi della propaganda per immagini si veda GIROTTO, Elisabetta, Politics and media, cit.; per approfondire l'universo dei colori politici si veda RIDOLFI, Maurizio, Italia a colori, cit.

${ }^{33}$ CASALINI, Maria, op. cit., p. 238.

${ }^{34}$ Sull'effetto indiretto di immagini pacificatrici, come "richiamo all'ordine" nei confronti di possibili atti di violenza in risposta alle provocazioni cattoliche cfr. CAVAZZA, Stefano, Comunicazione di massa e simbologia politica nelle campagne elettorali del secondo dopoguerra, in BALLINI, Pier Luigi, RIDOLFI Maurizio (a cura di), Storia delle campagne elettorali, Milano, Mondadori, 2002, pp. 193-237, p. 212.

${ }^{35}$ GIROTTO, Elisabetta, Politics and Media, cit.

${ }^{36}$ Sull'imitazione (sempre taciuta) di modelli organizzativi e formule propagandistiche del regime, cfr. VENTRONE, Angelo, Crisi della società e radicamento dei partiti di massa, in MICCOLI, Giovanni, et al. (a cura di), La grande cesura, cit., pp. 465-477; ID., Simboli e liturgie politiche nella propaganda del dopoguerra, in SALVATI, Mariuccia (a cura di), La fondazione della repubblica. Modelli e immaginario repubblicani in Emilia e Romagna negli anni della Costituente, Milano, Franco Angeli, 1999, pp. 159-183.

${ }^{37}$ PAJETTA, Gian Carlo, «Considerazioni sulla propaganda», in Quaderno dell'attivista, 10-11/1948, pp. 16-17.
} 
come una vera e propria «scampagnata»: la più tipica forma di svago delle famiglie proletarie. La stessa che il dopolavoro fascista aveva del resto ampiamente sperimentato in precedenza ${ }^{38}$.

L'assoluta garanzia del successo era poi affidata all'imitazione delle liturgie tipiche delle feste cattoliche, con i cortei che avrebbero visto sfilare i compagni in processione per rendere grazie alla figura «religiosa» del Segretario, mentre le immagini dei padri storici del comunismo sovietico venivano chiamati a surrogare le icone dei santi ${ }^{39}$. Il tutto condensato in un happening nel quale anche gli elementi del loisir, i sani piaceri della tavola e del ballo, gli spettacoli, lo sport, e persino il momento emozionante della lotteria (sempre a scopo sociale) sembrano assumere un aspetto rituale, destinato a scandire i tempi delle "sagre gioiose» in occasione delle quali $\mathrm{i}$ compagni si propongono di accogliere «gli amici di ogni tendenza politica, e anche gli incerti e gli avversari», che sarebbero senza dubbio stati conquistati dall'«invito sincero e dalla mano tesa» dei comunisti ${ }^{40}$.

E gli stessi irresistibili elementi di richiamo della festa annuale, finalizzati ad avvalorare la percezione collettiva del socialismo come sinonimo di serenità e a potenziare allo stesso tempo sia la visibilità delle capacità organizzative dei compagni che la forza di attrazione delle iniziative del partito, dovevano puntualmente ripresentarsi ogni volta che si trattava di mettere in agenda una iniziativa delle donne. Così la prima assise delle compagne, organizzata dal Pci dal 2 al 5 giugno 1945, come le feste parrocchiali, sarebbe stata animata da pesche di beneficenza con capi di abbigliamento, scarpe, stufette elettriche, libri e quaderni, oltre a polli, uova e possibilmente anche un maiale. A seguire naturalmente giostre, baracconi, tiro a segno, in un'aria pervasa dalle note dei canti partigiani, nella quale solo in ultimo sarebbero risuonate le parole dell'oratore di turno del partito (ancor meglio se in coppia con la compagna):

\begin{abstract}
Il comizio - osserva Maria Antonietta Macciocchi - era più rassicurante, meno traumatizzante con la donna e l'uomo comunisti insieme, poiché ricostruiva la gerarchia familiare e indirettamente riproponeva una tranquillizzante immagine della coppia. Coppia politica, ma pur sempre coppia. La donna per la strofetta iniziale, l'uomo per la sostanza. Se poi i due erano davvero sposati era l'apoteosi ${ }^{41}$.
\end{abstract}

\footnotetext{
${ }^{38}$ Scampanate, merende, giochi popolari, ciclismo, omaggio ai caduti, messa, poi giochi a premi col tiro alla fune e nel pomeriggio danze: questo il programma di una tipica festa dell'Ond: in proposito cfr. CAVAZZA, Stefano, Piccole patrie. Feste popolari tra regione e nazione durante il fascismo, Bologna, Il Mulino, 2003, p. 107.

${ }^{39}$ TONELLI, Anna, Falce e tortello. Storia politica e sociale delle feste dell'Unità (1945-2011), Roma, Laterza, 2012; sulla rappresentazione comunista si veda GIROTTO, Elisabetta, Politics and media, cit., pp. 165 et seq.

${ }^{40}$ PAJETTA, Gian Carlo, Considerazioni sulla propaganda, cit. p.16; sul tema si veda CASALINI, Maria, op. cit., p. 244; Sulla propaganda per immagini del Pci si veda: GIROTTO, Elisabetta, Politics and media, cit.

${ }^{41}$ MACCIOCCHI, Maria Antonietta, Duemila anni di felicità. Diario di un'eretica, Milano, Il Saggiatore, 2000, p. 200.
} 
L'anno successivo, il II Congresso dell'Udi - anch'esso concepito come una vera e propria «sagra di vita femminile» - sarebbe poi stato corredato, oltre che da cortei di compagne nei diversi costumi regionali, da una «mostra di arti femminili», con l'esposizione di «merletti, paglie, ricami, lavorazione di giocattoli», e animato da proiezioni cinematografiche, recite di belle commedie e gite sui laghi. L'organizzazione di scampagnate fuori porta è peraltro destinata a restare un elemento costitutivo di base dei meeting femminili, talvolta declinato in versione di «pellegrinaggio» verso mete simboliche, come la tomba di Maria Margotti: una delle sante laiche, «martire della lotta di classe», che insieme a Irma Bandiera, Anna Maria Enriquez Agnoletti e le sorelle Arduino, Togliatti reputava utile le italiane aggiungessero agli altarini domestici che amavano tanto custodire ${ }^{42}$.

A dimostrazione della natura sostanzialmente conciliatoria della linea politica comunista ancor più evidente sul fronte della propaganda femminile - il rito della messa era già stato incorporato nel programma del I Congresso nazionale dell'Udi e sarebbe stato puntualmente inserito in quello della celebrazione dell'8 marzo romano, corredato dagli immancabili rituali di omaggio alla memoria dei caduti e alle iniziative di beneficenza, ma anche da cene, tombolate, concerti e spettacoli di vario genere ${ }^{43}$. Se la ricorrenza della giornata internazionale della donna non era una novità, dal momento che già nel 1921 il Pci aveva indetto per l'occasione una serie di comizi, a rinnovarne la fisionomia sarebbe stata dunque ancora una volta la componente ricreativa: quell'"uso politico del loisir" che Togliatti aveva individuato come uno dei «bisogni primari della masse» cui il fascismo, a suo modo, aveva compreso la necessità di andare incontro ${ }^{44}$.

Anche le compagne, la cui presenza si confermava come un elemento essenziale di tutti i palcoscenici di riconoscimento collettivo del partito, avrebbero avuto così "una festa tutta per loro", nella cornice incantata di un tripudio di fiori e con tanto di «torta dell' 8 marzo» ${ }^{45}$ !

\footnotetext{
${ }^{42} \ll$ Vorrei si facessero a milioni delle immagini a colori di queste donne per distribuirle alle donne del popolo che le conservassero insieme alle immagini dei santi» - avrebbe affermato Togliatti dopo aver rilevato che gli unici modelli femminile forti che le italiane avevano di fronte a loro erano le sante (Caterina e Chiara). Era perciò opportuno ricreare nuove immagini "sacre" di donne eroiche, il cui monopolio non poteva essere lasciato alla Chiesa: cfr. TOGLIATTI, Palmiro, «L'emancipazione della donna: un problema centrale del rinnovamento dello Stato italiano e della società italiana, discorso pronunciato alla I Conferenza femminile del Partito comunista italiano», Roma, 2-5 giugno 1945, in ID., L'emancipazione femminile, Roma, Editori Riuniti, 1973, p. 34; in proposito cfr. LUSSANA, Fiamma, 1944-1945, Togliatti, cit., p. 161.

${ }^{43}$ Cfr. GISSI, Alessandra, Otto marzo. La giornata internazionale delle donne in Italia, Roma, Viella, 2010, p. 38; GABRIELLI, Patrizia, La pace e la mimosa. L'Unione donne italiane e la costruzione politica della memoria, (19441945), Roma, Donzelli, 2005, p. 61 et seq.

${ }^{44}$ CASALINI, Maria, op. cit., p. 245.

${ }^{45}$ VERGALLI, Teresa, Storie di una staffetta partigiana, Roma, Editori Riuniti, 2004, p. 265.
} 


\section{Un partito di mogli e mamme}

«Stelline dell'Unità», cuoche, compagne di ballo, propagandiste indefesse, le donne rivelavano i loro tanti volti - tutti funzionali alla causa - ma restavano comunque sempre soprattutto madri, per i comunisti come per i cattolici. Non a caso, ancor prima di diventare uno degli elementi portanti della propaganda di regime, l'esaltazione della maternità aveva rappresentato un tema tutt'altro che secondario della pedagogia sociale del Pci di Gramsci e Bordiga. «Bisogna che la società riconosca il ruolo della Madre [...]. Quando verrà abolito lo sfruttamento [...] alla maternità sarà riconosciuto il suo valore vero [...] sarà perciò circondata da tutte le cure [...], perché sacra $\mathrm{e}$ degna di religioso rispetto è la madre», si leggeva ad esempio su "L'Ordine nuovo» ${ }^{46}$. E persino nella Russia rivoluzionaria, dove la Kollontaj si faceva paladina del libero amore, alla maternità era riconosciuto un ruolo sociale essenziale.

È dunque sull'onda lunga della continuità con la tradizione terzinternazionalista della prima ora, oltre che in tacito accordo con le linee guida dalla politica sociale del fascismo e in ottemperanza dei dettami della Casti Connubii, che la figura della madre è destinata ad assumere un ruolo chiave nel linguaggio del partito nuovo. A testimoniarlo basterebbe l'elenco dei compiti affidati alle donne sedute sui banchi della Sinistra all'Assemblea Costituente: chiamate a «rappresentare le famiglie italiane e le loro difficoltà di sussistenza», e incaricate di rivendicare i diritti della donna come madre, ancor prima che come lavoratrice e cittadina ${ }^{47}$. Per questo l'appeal dell'immagine della candidate del Pci in sede di campagna elettorale era stato tutto incentrato sul loro ruolo domestico e soprattutto sulla loro funzione materna ${ }^{48}$. Così recita, ad esempio la scheda biografica di Marisa Rodano apparsa su «L’Unità»

È innamoratissima della vita domestica e qualche volta quasi rimpiange di doversene distaccare diverse ore al giorno per il suo lavoro al Comune e all'Udi: ma poi dice a se stessa che, appunto perché è una mamma, il dovere la chiama laddove c'è da fare qualcosa in favore della altre mamme [...]. E allora scaccia la tentazione: depone il lavoro che sta sferruzzando, il golfino nuovo di Paoletta, e va a vedere che cosa si può fare per appoggiare le donne di una borgata che sono in agitazione ${ }^{49}$.

Grassa, con i capelli lunghi e diversi figli, rappresentava proprio la candidata ideale del Pci avrebbe ammesso la stessa Rodano ${ }^{50}$; e Nella Marcellino, con altrettanta autoironia, ricorda come

\footnotetext{
${ }^{46} \mathrm{Cfr}$. L'Ordine nuovo, 3 marzo 1921.

${ }^{47}$ «Il programma dei lavori del I Congresso dell'Udi», in Noi donne, 15 ottobre 1945.

${ }^{48}$ CASALINI, Maria, op. cit., pp. 194-195.

${ }^{49}$ MESSINA, C., «Le vostre candidate», in L'Unità, 14 marzo 1948. Cit. in CASALINI, Maria, op. cit., p. 195.

${ }^{50}$ Cfr. MAFAI, Miriam, op. cit., p. 148.
} 
fosse stata costretta per anni a raccontare una serie di bugie, per tacitare le continue domande sulla sua vita privata da parte dei compagni, che non sapevano farsi una ragione del perché lei e Arturo Colombi non avessero avuto figli ${ }^{51}$. Anche nella loro libreria, del resto, come in quella di qualsiasi compagno che si rispettasse, troneggiava una copia de La Madre di Gorkij.

Non può quindi di certo destare meraviglia il fatto che la proposta di legge sulla quale il Pci di Togliatti pensava di imperniare la propria popolarità fra le masse, fosse destinata a tutelare la maternità. Se il tono della campagna di propaganda si differenzia del resto dalla retorica demografica del regime per assumere toni più vicini a quella cattolica, ne mantiene tuttavia l'ispirazione di fondo, ampliandone l'applicazione in senso universalistico.

Con ragione Maria Casalini sostiene che insieme a quella della madre, nel dopoguerra, sarebbe stata la figura della casalinga ad assumere una inedita centralità nell'universo mentale degli italiani, soprattutto, ma non solo, a seguito della martellante esaltazione dei cattolici della missione domestica della donna. Così, la sostanziale novità che la proposta di legge Noce-Di Vittorio finiva col presentare rispetto alla legislazione del 1934 era quella di estendeva l'area dell'assistenza riservata alle operaie, alle contadine e soprattutto alle casalinghe, inserite a pieno titolo all'interno della categoria delle «lavoratrici» nel testo da sottoporre all'approvazione del Parlamento ${ }^{52}$.

Per il resto, a motivare il rafforzamento della prassi "protettiva" delle madri sarebbe stato accampato ancora una volta il tema della "tutela della stirpe». All'interrogativo: "perché le mamme domandano [una più ampia legislazione di tutela]» la risposta - corroborata da una dettagliata sequenza di dati - prospettata dal "Quaderno dell'attivista» è: "perché i bimbi muoiono soprattutto nel primo anno di età? Perché i bimbi muoiono soprattutto quando le mamme non possono allattarli $»^{53}$. Per questo, insieme al «premio di natalità» Teresa Noce proponeva di inserire il "premio di allattamento», e sul tema dell'“educazione alla maternità" si portava avanti una vasta campagna d'informazione, mentre stampa, comizi e giornali murali riportavano puntualmente la cronaca dell'iter parlamentare della proposta di legge comunista e ne illustravano i dettagli ${ }^{54}$.

L'impressione, insomma, è che si importino modelli sperimentati di propaganda per adattarli a contenuti nuovi, facendo tesoro della quota di consenso che avevano garantito al regime. Sono del

\footnotetext{
${ }^{51}$ LUNADEI, Simona, MOTTI, Luisa, RIGHI, Maria Luisa (a cura di), È brava ma... Donne nella CGIL 1944 1962, Roma, Ediesse, 1999, p. 190.

${ }^{52}$ CASALINI, Maria, op. cit., p. 211.

${ }^{53}$ «Perché le mamme domandano», in Quaderno dell'attivista, 10/1947, p. 3; "Campagne in corso», in Quaderno dell'attivista, 9-10/1947; "Campagna nazionale per la tutela della maternità», in Quaderno dell'attivista, 8/1948, p. 16.

${ }^{54}$ NOCE, Teresa, «La maternità funzione sociale», in Vie nuove, 22 settembre 1946. «Pur non mirando al numero come fattore di potenza, dobbiamo preoccuparci che il bilancio demografico, divenuto in alcune circoscrizioni negativo, si mantenga tale: sarebbe il suicidio della nazione»: cfr. «La lotta contro la mortalità infantile», in Rinascita, 5-6/1946, pp. 27-32.
} 
resto le parole stesse di Togliatti, alla ricerca di strumenti di persuasione capaci di ampliare l'area di influenza al partito, soprattutto fra le contadine al Sud, a testimoniare come le organizzazioni di massa del regime avrebbero rappresentato un esempio da seguire. «Bisogna studiare quello che erano le "Massaie rurali" - avrebbe esplicitamente ammesso nel corso di una riunione della Direzione - e prendere quel tanto di positivo che vi era in tale organismo» ${ }^{55}$. Né meno esplicito, sul fronte della continuità con la pratica della capitalizzazione del consenso garantito dalle pratiche assistenziali sperimentate dal fascismo, appare l'avvio della campagna per i prestiti matrimoniali.

Ideata dal nazismo nel 1933, allo scopo dichiarato di disincentivare l'occupazione femminile, a partire dal 1937 l'iniziativa dell'elargizione del prestito di una somma di denaro alle coppie di giovani sposi era stata importata in Italia dal fascismo, in declinazione pronatalista ${ }^{56}$. E la stessa iniziativa sarebbe stata riesumata da Laura Diaz, prima firmataria con Giuliana Nenni, Gina Fanoli, Nadia Spano, Luciana Viviani, della proposta di legge presentata in Parlamento nel settembre del $1948^{57}$.

A raccogliere la quota più consistente di consenso popolare sarebbe stata poi la vasta campagna di propaganda dell'iniziativa intrapresa, in nome dell'Associazione ragazze italiane (ARI), da Marisa Musu, con un ampio dispendio di retorica rituale.

Così, in parallelo con la «Giornata della famiglia e della Pace», sarebbe stata indetta, l'anno successivo, la «Festa del prestito», seguita dalla «Giornata delle delegazioni » di giovani aspiranti sposine che si sarebbero dovute dirigere verso le Prefetture delle diverse città e reclamare il proprio diritto al matrimonio, per culminare infine nella «Giornata del telegramma», fissata il 24 marzo 1949. In quel giorno il partito chiedeva una vera e propria mobilitazione al femminile; le giovani sposine dovevano a spedire un telegramma all'onorevole Gronchi, allora presidente della Camera, per sollecitare la proposta di legge che veniva incontro alle esigenze primarie dei giovani sposi $^{58}$. Secondo Casalini

l'urgenza del provvedimento, era motivata della legittima protesta nei confronti della «condanna al celibato» perpetrata dal governo De Gasperi nei confronti di tanti giovani

\footnotetext{
${ }^{55}$ Verbale della riunione della Direzione del 17 gennaio 1945, intervento di Ercoli, p. 2, in FIG, APC, MF 272, Verbali della Direzione, 1945. Sulle massaie rurali cfr. WILLSON, Perry, «Contadine e politica nel Ventennio. Le Sezione massaie rurali», in Italia contemporanea, 218, 2000, pp. 31-47.

${ }^{56}$ SALVANTE, Martina, «I prestiti matrimoniali: una misura pronatalista nella Germania nazista e nell'Italia fascista», in Passato e presente, XXI, 60, 3/2003, pp. 39-58.

${ }^{57} \mathrm{Cfr}$. la proposta di legge, corredata peraltro di un preciso conteggio delle spese che una giovane coppia avrebbe dovuto sostenere per mettere su casa (affitto, mobili, piatti, pentole posate, lenzuola, trapunta, tovaglie, federe, asciugamani e così via), in ATTI PARLAMENTARI, Camera dei Deputati, Documenti. Disegni di legge e relazioni. Proposta di legge n. 113, 29 settembre 1948, Prestiti matrimoniali.

${ }^{58}$ CESARINI, G., «Che cosa sognano i nostri giovani?», in Noi donne, 17 aprile 1949.
} 
proletari desiderosi di farsi una famiglia, veniva giustificata dalle allarmanti statistiche riguardo al numero dei matrimoni e soprattutto al declino delle nascite ${ }^{59}$.

La tattica reputata vincente, in altre parole, appare quella della ritorsione delle accuse: non è il Partito comunista, che ha a cuore il benessere delle giovani coppie, oltre che di tutte le "mamme" italiane e dei loro figli, ma la Democrazia cristiana che distrugge le famiglie italiane, sia impedendo a quelle nuove di nascere che gettando le altre nell'abisso della miseria ${ }^{60}$. E il compito di metterla in pratica, tanto arduo quanto essenziale per gli equilibri politici del paese, il Pci lo delegava, evidentemente, alle donne.

\section{Vola colomba}

In realtà, l'attività femminile si articola su un doppio binario. Da un lato, come abbiamo visto, c'era la necessità di monitorare da vicino le esigenze più immediate della gente; e per essere sicuri di concentrare i propri sforzi sui punti nevralgici del malessere diffuso alla base era necessario seguire la strategia del "porta a porta". Visto che la premessa della buona riuscita di quella che Luciana Viviani ha definito «la politica del quotidiano» era la percezione esatta delle impellenze concrete, caso per caso, delle famiglie italiane, all'interno delle famiglie stesse era necessario aprirsi un varco ${ }^{61}$. Se qualsiasi estraneo sarebbe stato accolto quanto meno con diffidenza, una chiacchierata con la vicina di casa, che magari invitava le amiche e le conoscenti per un caffè, poteva rappresentare la soluzione migliore per entrare in contatto con le massaie. Per questo si sarebbe pensato di ricorrere alla formula delle «riunioni di caseggiato» gestiti da una compagna dotata di verve e spigliatezza, addestrata a rivestire il ruolo della «comare». A questa vasta opera di monitoraggio avrebbe poi fatto seguito la creazione di un numero inverosimile di comitati rionali e di organizzazioni di tutti i tipi, regionale e locale, come le «Mamme napoletane», le «Mogli degli emigranti», le «Donne della Montagna», le «Mogli dei carcerati» e le «Mogli dei Minatori». Insistentemente la direzione del Pci sottolineava come le donne dovessero avere «un obiettivo concreto», e tutto ciò che era legato ai bisogni della casa e della famiglia risultava centrale $^{62}$. Così buona parte degli sforzi dell'Udi, a fine degli anni Quaranta, si sarebbe concentrata nell'ulteriore incremento del numero del «gruppi specifici». Oltre alle donne capofamiglia, nasceranno così il «Gruppo Casalinghe», le «Donne di Campagna», le «Amiche della Scuola», le

\footnotetext{
${ }^{59}$ CASALINI, Maria, op. cit., p.194.

${ }^{60}$ «Le mamme romane voteranno per (Nadia Gallico Spano e Marcella Ferrari Lapiccirella)», in L'Unità, 16 maggio 1946.

${ }^{61}$ Cfr. ROSSI DORIA, Anna, Le donne sulla scena politica, in Storia dell'Italia repubblicana, vol. I, La costruzione della democrazia. Dalla caduta del fascismo agli anni cinquanta, Torino, Einaudi, 1994, p. 793 et seq.

${ }^{62}$ CASALINI, Maria, op. cit., pp. 140-141.
} 
«Ragazze d'Italia», il «Gruppo di lettrici e diffonditrici di Noi Donne», le «Amiche dell' infanzia», ma anche più fantasiosi «Gruppi turistici», 0 «Circoli della Moda» ${ }^{63}$.

A complemento di tutte queste iniziative di base, a partire dal 1947 prenderà d'altra parte il via una grande battaglia unitaria, che tutte le donne avrebbero dovuto combattere in formazione compatta, sotto le insegne del pacifismo. Com'era avvenuto in occasione della guerra di Libia, la cultura marxista avrebbe assegnato loro l'etichetta di «combattenti per la pace», e l'impegno in tal senso balza immediatamente al primo posto tra le mansioni affidate dal partito alle compagne, ancor prima sia della campagna in favore della legge sulla maternità che della lotta contro il carovita e l'assistenza invernale ${ }^{64}$. D'altro canto

per l'occasione ad essere chiamata in ballo è dunque anche l'eredità delle tradizioni del movimento operaio, generalmente assai poco sensibile alla causa del proletariato femminile, ma altrettanto solerte nel sollecitarne la discesa in campo quando si era trattato di contrastare la politica espansionistica di Giolitti ${ }^{65}$.

In armonia con le stesse direttive della linea staliniana in tema di organizzazioni di massa anche se in aperta controtendenza rispetto all'acuirsi delle tensioni sul fronte interno l'ispirazione del collateralismo femminile si conferma ancora una volta aclassista, unanimistica e solidaristica. Rispetto a trent'anni prima il loro impegno organizzativo appare del resto centuplicato. Mentre in passato il Psi si era limitato ad alimentare la stampa e a fomentare moti spontanei di protesta, le «Amiche della Pace» avrebbero dato vita adesso ad una serie di iniziative della più varia natura, dando dimostrazione di una creatività a dir poco esuberante. In occasione delle date di un apposito Calendario della Pace, avrebbero così instancabilmente promosso pellegrinaggi verso i luoghi della memoria, conferenze e manifestazioni di vario genere ${ }^{66}$. A Torino, ad esempio, avrebbero collocato 12 cassette giganti nelle quali i bambini erano invitati ad imbucare le loro letterine per la pace al Presidente della Repubblica, mentre a Milano si organizza una grande manifestazione al teatro Oberdan, dove sono sempre invitati ad intervenire tanti bimbi, tutti con il loro «raccontino per la pace» in una mano e il palloncino della pace nell'altra ${ }^{67}$. Ovunque le compagne avrebbero naturalmente indetto migliaia di assemblee ispirate

\footnotetext{
${ }^{63}$ Cfr. «Lo Statuto dell'Udi», in Bollettino d'informazione, ottobre 1949. Vedi anche MICHETTI, Maria, REPETTO, Margherita, VIVIANI, Luciana, op. cit., pp. 322-323.

${ }^{64}$ Cfr. Circolare n. 11. Orientamenti per il lavoro femminile, in «Istruzioni e direttive di lavoro della Direzione del Pci a tutte le Federazioni», in supplemento al Quaderno dell'attivista, 9-10 settembre 1947, pp. 21-22.

${ }^{65}$ Cfr. CASALINI, Maria, «I socialisti e le donne. Dalla "mobilitazione pacifista" alla smobilitazione postbellica», in Italia contemporanea, 222, 2001, pp. 5-41, in particolare pp. 9-10.

${ }^{66}$ "Appello alle donne italiane approvato al II Congresso nazionale delle Donne d'Italia», in Noi donne, 1 novembre 1947 e in Bollettino d'informazione, novembre 1947.

${ }^{67}$ In Bollettino d'informazione, 1 gennaio 1949.
} 
all'esaltazione del dolore delle vedove, delle madri, delle vittime della guerra e organizzato «Giornate della famiglia e della Pace». Ma soprattutto sarebbero state impegnate a montare banchetti in tutte le piazze d'Italia per la raccolta delle firme contro l'atomica e a cucire altrettante migliaia di bandiere per la pace: quelle stesse bandiere che in epoca risorgimentale avevano cucito per la patria. Perché dell'appello ad una sorta di "ennesimo Risorgimento" doveva trattarsi, combattuto contro un paese manovrato dai «banchieri e dai mercanti di bombe atomiche americani», e pronto allo stesso tempo a raccogliere il testimone delle spirito patriottico delle guerre d'Indipendenza, di cui la Resistenza era stata la prima erede ${ }^{68}$. Così l'immagine di Garibaldi come simbolo del Fronte delle sinistre viene sapientemente abbinato al richiamo dell'esperienza resistenziale nella definizione che sarebbe stata scelta per l'intero movimento: quella di Partigiani della Pace ${ }^{69}$. Uno dei tanti ossimori che dell'identità del partito nuovo riflettono la natura più profonda.

Il fine elettorale emerge con immediatezza dalla campagna elettorale del 1948, significativa infatti la copertina del numero di «Noi donne» del primo marzo 1948: un'immagine femminile con in mano una colomba bianca, una futura icona per le prossime elezioni ${ }^{70}$. Ma nel movimento dei Partigiani della Pace - battezzato come tale nel 1949 ma ampiamente anticipato negli anni precedenti da una fitta serie di iniziative - erano in ballo finalità più ampie. Tanto che la campagna avrebbe subito una prima forte accelerazione proprio dopo la sconfitta del 18 aprile per trarre in seguito nuovo impulso dall'attentato a Togliatti, fino a raggiungere il climax con la formazione della Nato. Poche iniziative di propaganda, in realtà, appaiono più multiformi e complesse. Nel movimento per la pace si intrecciano infatti temi di politica interna e di politica estera, e si racchiudono obiettivi a breve e a lunga scadenza. Così la tematica antiamericana si salda a quelle dell'indipendenza nazionale e della lotta contro il "governo della guerra civile» forte della violenza della polizia di Scelba ${ }^{71}$, mentre alla «politica guerrafondaia» made in USA si contrappone l'immagine del paese democratico e antimilitarista per eccellenza, dove dominano serenità, benessere, e un «appassionato amore per la pace nel mondo» ${ }^{72}$. Poco importa, in fondo, se l'immagine che si fornisce dell'URSS si rivela del tutto illusoria; fatto sta che sono i valori della solidarietà universale che si vogliono affermare nella nuova repubblica ${ }^{73}$. Per questa via, per quanto il movimento sia ben lungi dal riflettere l'ispirazione super partes che si tende ad

\footnotetext{
${ }_{68}^{68}$ «Donne, il Pci è il vostro partito», in Propaganda, 20 gennaio-5 febbraio 1948.

${ }^{69}$ In proposito cfr., tra gli altri, PETRANGELI, Giulio, «I Partigiani della pace in Italia 1948-1953», in Italia contemporanea, 217, 1999, pp. 667-692; CERRAI, Sandra, I Partigiani della Pace. Tra utopia e sogno egemonico, Padova, Edizioni Libreria universitaria, 2011.

${ }^{70}$ Sul rapporto donne e elezioni si veda CASALINI, Maria, Le donne della sinistra, cit., p. 241.

${ }^{71}$ «Una nuova lotta per la pace e la libertà», in Propaganda, 20 agosto 1948.

${ }^{72}$ MICHELETTI, Rita, «Di ritorno dall'URSS. Le ragazze raccontano», in Noi Donne, 1 settembre 1946.

${ }^{73}$ BALLONE, Adriano, Il militante comunista torinese (1945-1955). Fabbrica, società e politica: una prima ricognizione, in AGOSTI, Aldo (a cura di), I muscoli della storia. Militanti e organizzazioni operaie a Torino 1945-1955, Milano, Franco Angeli, 1987, pp. 121-130.
} 
attribuirgli a livello propagandistico, gli obiettivi della politica estera sovietica finiscono col subire una vera e propria trasmutazione, assumendo la fisionomia di uno strumento di autentica ispirazione umanitaria. Un vero e proprio esempio dell'eterogenesi dei fini! Ed è qui che il contributo delle donne attribuisce alla campagna un essenziale valore aggiunto. La massiccia mobilitazione femminile, sul piano internazionale, fornisce infatti le migliori garanzie di credibilità ad un'iniziativa che altrimenti rischiava di rivelare troppo scopertamente le proprie finalità strumentali. Chi poteva sospettare in altre parole che le donne del mondo si fossero incontrate a Parigi se non per affermare con slancio e sincerità quella volontà di pace che corrispondeva alla loro più intima natura? Poco importa se la storia aveva dimostrato la totale infondatezza dello stereotipo. La donna avrebbe comunque continuato a incarnare nell'immaginario dell'Occidente il simbolo vivente della guerra alla guerra.

D'altro canto con il sovrappiù dell'esibizione dei più bei nomi dell'intellighenzia «democratica» internazionale, da Amado ad Aragon, da Heinrich Mann a Pablo Neruda, sotto la magica insegna della Paloma di Picasso, Togliatti poteva davvero pensare di avere scelto la strada giusta per allargare la propria sfera di consenso nel paese, facendo appello ai sentimenti più radicati dell'animo popolare, condivisibili anche dall'elettorato cattolico ${ }^{74}$. Dopo aver presentato tali e tante credenziali, come si poteva continuare a credere all'affidabilità dei manifesti democristiani che continuavano a rappresentare i comunisti nelle vesti del «lupo famelico malcelato sotto le spoglie dell'agnello» ${ }^{75}$ ?

\footnotetext{
${ }^{74}$ CASALINI, Maria, Le donne della sinistra, cit., p. 247.

${ }^{75}$ SASSOON, Donald, Togliatti e la via italiana al socialismo. Il Pci dal 1944 al 1964, Torino, Einaudi, 1980, pp. 99, 112.
} 


\section{L'AUTORE}

Elisabetta GIROTTO è ricercatrice all'IHC dell'Università Nova di Lisbona, I suoi principali campi d'interesse sono la storia della famiglia fra il 1930 e il 1970 e la storia delle generazioni e dei mezzi di comunicazione di massa.

URL: < http://www.studistorici.com/progett/autori/\#Girotto > 